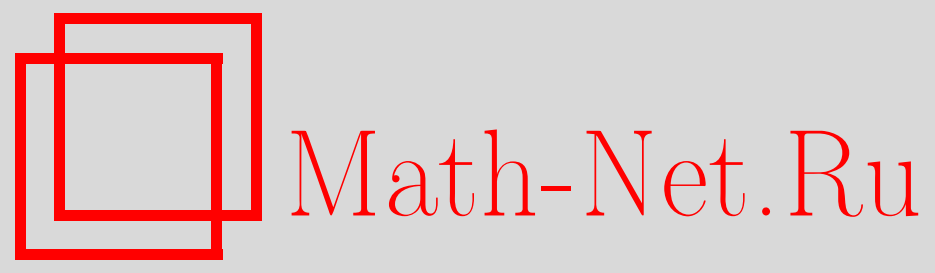

А. М. Козлов, О вероятностях больших уклонений статистики Шеппа, Дискрет. матем., 2004, том 16, выпуск $1,140-145$

DOI: https://doi.org/10.4213/dm148

Использование Общероссийского математического портала Math-Net.Ru подразумевает, что вы прочитали и согласны с пользовательским соглашением http://www . mathnet.ru/rus/agreement

Параметры загрузки:

IP : 54.210 .77 .194

26 апреля 2023 г., 17:40:50 


\title{
О вероятностях больших уклонений статистики Шеппа
}

\author{
() 2004 г. А. М. Козлов
}

Для статистики Шеппа, равной максимуму колебаний случайного блуждания $S_{n}=\sum_{i=1}^{n} \xi_{i}$ в окне ширины $L$, когда это окно перемещается в пределах отрезка $[1,2 L]$, получена асимптотика при $L \rightarrow \infty$ вероятности больших уклонений $\mathbf{P}\left(W_{L, L} \geqslant \theta L\right)$, где $\theta$ - постоянная. Предполагается, что $\xi_{1}, \xi_{2}, \ldots$ - независимые одинаково распределенные случайные величины с нерешетчатым распределением, удовлетворяющим правостороннему условию Крамера. Показано, что асимптотика имеет вид $H_{\theta} L \mathrm{P}\left(S_{L} \geqslant \theta L\right)$, где $H_{\theta}$ - зависящая от $\theta$ постоянная, $0<H_{\theta}<1$.

Работа выполнена при поддержке Российского фонда фундаментальных исследований, проект 01-0100-649.

Классическая теория больших уклонений для сумм

$$
S_{n}=\sum_{i=1}^{n} \xi_{i}
$$

независимых одинаково распределенных случайных величин при выполнении (правостороннего) условия Крамера $[1,2]$

$$
R(h)=\int_{-\infty}^{\infty} e^{h x} d F(x)<\infty, \quad 0 \leqslant h<b \leqslant \infty, \quad F(x)=\mathbf{P}\left(\xi_{1} \leqslant x\right),
$$

была распространена в работах [3-5] на статистику Эрдеша-Реньи

$$
T_{n, m}=\max _{1 \leqslant k \leqslant n} \eta_{k, m}, \quad \eta_{k, l}=\sum_{i=k}^{k+l-1} \xi_{i}
$$

Исследовался также вопрос о больших уклонениях для статистики Шеппа

$$
W_{n, m}=\max _{1 \leqslant k \leqslant n} \max _{1 \leqslant l \leqslant m} \eta_{k, l}
$$

в случае стандартного гауссовского распределения случайных величин $\xi_{1}, \xi_{2}, \ldots$ В [3-5] так же, как и в настоящей статье, используется так называемый метод двойных сумм, в 
котором известные результаты о больших уклонениях сумм $S_{n}$ совмещены с методологией, развитой в теории экстремумов гауссовских процессов и полей [6]. В настоящей работе при $L \rightarrow \infty$ получена асимттотика

$$
\mathbf{P}\left(W_{L, L} \geqslant \theta L\right) \sim \sqrt{L / 2 \pi}\left(H_{\theta} /\left(h_{\theta} \sigma\left(h_{\theta}\right)\right) e^{-\Lambda(\theta) L},\right.
$$

где распределение вероятностей случайных величин $\xi_{1}, \xi_{2}, \ldots$ предполагается нерешетчатым и использованы обозначения теории больших уклонений сумм $S_{n}$ при выполнении условия Крамера (1). Здесь и далее

$$
m(h)=R^{\prime}(h) / R(h), \quad \sigma^{2}(h)=m^{\prime}(h)>0, \quad a=\lim _{h \rightarrow b-} m(h),
$$

$h_{\theta}$ - единственный корень уравнения $m(h)=\theta, 0<\theta<a, H_{\theta}$ - зависящая от $\theta$ постоянная, $0<H_{\theta}<1$,

$$
\begin{aligned}
\Lambda(\theta) & =h_{\theta} \theta-\ln R\left(h_{\theta}\right), \quad 0<\theta<a, \\
\pi(L ; \theta) & =\left(\sqrt{2 \pi L} h_{\theta} \sigma\left(h_{\theta}\right)\right)^{-1} e^{-\Lambda(\theta) L} .
\end{aligned}
$$

Асимптотика вероятностей больших уклонений при $L \rightarrow \infty$ имеет в случае нерешетчатого распределения $F$ вид [1]

$$
\mathbf{P}\left(S_{L} \geqslant \theta L\right)=\pi(L ; \theta)(1+o(1)) .
$$

В [8] получено неравенство (см. также (41) в [5])

$$
\mathbf{P}\left(S_{L} \geqslant \theta L, S_{L+r}-S_{r} \geqslant \theta L\right) \geqslant 2 q_{\theta}^{r} P\left(S_{L} \geqslant \theta L\right), \quad 1 \leqslant r \leqslant L,
$$

при некотором $0<q_{\theta}<1$. Нам потребуется следующее его обобщение.

Лемма 1. Пусть $\alpha, \beta, \gamma-$ некоторые натуральные числа, $\alpha+\beta \leqslant L, \beta+\gamma \leqslant L$. Тогда для некоторого $0<p_{\theta}<1$

$$
\mathbf{P}\left(S_{\alpha+\beta} \geqslant \theta L, S_{\alpha+\beta+\gamma}-S_{\alpha} \geqslant \theta L\right) \leqslant c p_{\theta}^{L-\beta} \mathbf{P}\left(S_{L} \geqslant \theta L\right),
$$

где с- постоянная.

Теорема 1. Пусть $\xi_{i}, i=1,2, \ldots$, - независимые одинаково распределенные случайные величины, для которых выполнено условие Крамера (1) и М $\xi_{1}=0$. Тогда в случае нерешетчатого распределения случайных величин $\xi_{1}, \xi_{2}, \ldots$ при $L \rightarrow \infty$

$$
\mathbf{P}\left(W_{L, L} \geqslant \theta L\right) \sim H_{\theta} L \pi(L ; \theta)
$$

равномерно по $\theta, 0<\varepsilon \leqslant \theta \leqslant a-\varepsilon$.

Теорема 2. В предположениях теоремы 1 при $L \rightarrow \infty$ равномерно по $\theta$ в указанных там пределах

$$
\mathbf{P}\left(W_{N, L} \geqslant \theta L\right) \sim H_{\theta} N \pi(L ; \theta)
$$

при $N$, стремячихся к бесконечности таким образом, что правая часть в (5) стремится к нулю, то есть при $N=o\left(\sqrt{L} e^{\Lambda(\theta) L}\right)$ при $L \rightarrow \infty$. 
Замечание 1. В решетчатом случае в (4), (5) добавляется множитель $d_{\theta}$.

Доказательство теоремы 1. Разобьем целочисленный квадрат $[1, L] \times[1, L]$ на квадраты размера $n \times n$ и положим

$$
\Delta_{i j}^{(n)}=((i-1) n, i n] \times(L-j n, L-(j-1) n], \quad i, j=1,2, \ldots,
$$

где нумерация по переменному $j$ выбрана с той целью, чтобы значению $j=1$ отвечали наибольшие дисперсия поля $\left(\eta_{k l}\right)$. Будем полагать при доказательстве число $L / n$ целым, поскольку областями разбиения, соответствующими крайнему значению $j=[L / n]+1$ можно пренебречь. Положим

$$
A_{i j}^{(n)}=\left\{\max _{k, l \in \Delta_{i j}^{(n)}} \eta_{k l} \geqslant \theta L\right\}, \quad C_{L n}^{\prime}=\bigcup_{i=1}^{L / n} A_{i 1}^{(n)}, \quad C_{L n}^{\prime \prime}=\bigcup_{i=1}^{L / n} \bigcup_{j=2}^{L / n} A_{i j}^{(n)},
$$

так что $\left\{W_{L, L} \geqslant \theta L\right\}=C_{L n}^{\prime} \cup C_{L n}^{\prime \prime}$. В основе доказательства теоремы лежат неравенства

$$
\sum_{i} \mathbf{P}\left(A_{i 1}^{(n)}\right)+\mathbf{P}\left(C_{L n}^{\prime \prime}\right) \geqslant \mathbf{P}\left(W_{L, L} \geqslant \theta L\right) \geqslant \sum_{i} \mathbf{P}\left(A_{i 1}^{(n)}\right)-\sum_{i<j} \mathbf{P}\left(A_{i 1}^{(n)} A_{j 1}^{(n)}\right) .
$$

Представим случайные величины $\eta_{k, l}$ при $(k, l) \in \Delta_{11}^{(n)}$ (то есть при $1 \leqslant k \leqslant n$, $L-n+1 \leqslant l \leqslant L)$ в виде

$$
\begin{aligned}
\eta_{k, l} & =\eta_{k, n-\dot{k}+1}+\eta_{n+1, L-2 n}+\eta_{L-n+1, k+l-L+n-1} \\
& \stackrel{d}{=} \eta_{k, n+r}+\eta_{k+n+r, L-2 n}, \quad r=l-L+n, \quad 1 \leqslant r \leqslant n .
\end{aligned}
$$

Положим

$$
G_{n}(y)=\mathbf{P}\left(\max _{1 \leqslant k, r \leqslant n} \eta_{k, n+r} \leqslant y\right), \quad \bar{G}_{n}(y)=1-G_{n}(y)
$$

и представим вероятность $\mathrm{P}\left(A_{11}^{(n)}\right)$ в виде

$$
\mathbf{P}\left(A_{11}^{(n)}\right)=\int_{-\infty}^{\infty} \mathbf{P}\left(S_{L-2 n} \geqslant \theta L-y\right) d G_{n}(y)
$$

Предполагая, что $n$ фиксировано, а $L$ стремится к бесконечности, найдем асимптотику вероятности (8). Разобьем действительную прямую на промежутки $\left(-\infty,-L^{1 / 3}\right)$, $\left[-L^{1 / 3}, L^{1 / 3}\right]$ и $\left(L^{1 / 3}, \infty\right)$ и обозначим $I_{1}^{(n)}, I_{2}^{(n)}, I_{3}^{(n)}$ интегралы (8) по соответствующим промежуткам. Применением техники работы [5] показывается, что при $L \rightarrow \infty$

$$
\begin{aligned}
& I_{2}^{(n)} \sim H_{\theta}^{(n)} R\left(h_{\theta}\right)^{-2 n} \mathrm{P}\left(S_{L} \geqslant \theta L\right), \\
& I_{1}^{(n)}+I_{3}^{(n)}=o\left(I_{2}^{(n)}\right), \\
& H_{\theta}^{(n)}=M \exp \left\{h_{\theta} \max _{1 \leqslant r, k \leqslant n} \eta_{k, n+r}\right\}=\int_{-\infty}^{\infty} e^{h_{\theta} y} d G_{n}(y) .
\end{aligned}
$$

Таким образом, при $L \rightarrow \infty$

$$
\mathbf{P}\left(A_{11}^{(n)}\right) \sim \mathbf{P}\left(S_{L} \geqslant \theta L\right) H_{\theta}^{(n)} R\left(h_{\theta}\right)^{-2 n} .
$$


Для оценивания вероятностей $\mathbf{P}\left(A_{1 j}^{(n)}\right), j \geqslant 2$, используется тот же подход, что и выше в случае $j=1$. При $(k, l) \in \Delta_{1 j}^{(n)}, j \geqslant 2$, представление (7) заменяется следующим:

$$
\eta_{k, l} \stackrel{d}{=} \eta_{k, n+r}+\eta_{k+n+r, L-(j+1) n}, \quad r=l-L+j n,
$$

где ввиду того, что $L-j n<l \leqslant L-(j-1) n$, справедливы неравенства $1 \leqslant r \leqslant n$. В результате вместо (8) получаем равенство

$$
\mathbf{P}\left(A_{1 j}^{(n)}\right)=\int_{-\infty}^{\infty} \mathbf{P}\left(S_{L-(j+1) n} \geqslant \theta L-y\right) d G_{n}(y) .
$$

Окончательная оценка приобретает вид

$$
\mathbf{P}\left(C_{L, n}^{\prime \prime}\right) \leqslant \sum_{j=2}^{L / n} P\left(A_{1 j}^{(n)}\right) \leqslant c_{\theta}(L / n) \mathbf{P}\left(S_{L} \geqslant \theta L\right) H_{\theta}^{(n)} R\left(h_{\theta}\right)^{-3 n}
$$

Займемся оценкой вероятности

$$
\mathbf{P}\left(A_{11}^{(n)} A_{21}^{(n)}\right) \leqslant \sum_{(k, l) \in \Delta_{1}^{(n)},(r, s) \in \Delta_{2}^{(n)}} \mathbf{P}\left(\eta_{k, l} \geqslant \theta L, \eta_{r, s} \geqslant \theta L\right)
$$

Очевидно, что общий член суммы (13) есть функция переменных $\alpha=r-k$, $\beta=\min (k+l, r+s)-r, \gamma=r+s-k-l$. Мы обозначим ее $\rho(\alpha, \beta, \gamma)$. Таким образом, правую часть неравенства (13) можно записать в виде

$$
\sum_{k=1}^{n} \sum_{r=n+1}^{2 n} \sum_{l=L-n+1}^{L} \sum_{s=L-n+1}^{L} \rho(\alpha, \beta, \gamma) .
$$

В (14) просуммируем отдельно слагаемые, для которых имеет место неравенство $r+s \leqslant k+l$, и обозначим эту сумму для краткости $\Sigma_{1}$; сумму (14), взятую по дополнительной области $r+s>k+l$, обозначим через $\Sigma_{2}$. Члены суммы $\Sigma_{1}$ оценим следующим образом:

$$
\mathbf{P}\left(\eta_{k, l} \geqslant \theta L, \eta_{r, s} \geqslant \theta L\right) \leqslant \mathbf{P}\left(\eta_{r, s} \geqslant \theta L\right) \leqslant c_{\theta}^{\prime} \mathbf{P}\left(S_{L} \geqslant \theta L\right) R\left(h_{\theta}\right)^{s-L},
$$

где $c_{\theta}^{\prime}$ - зависящая от $\theta$ постоянная. Воспользуемся представлением

$$
L-s=(n-k)+(r-n)+(L-l)+(k+l-r-s)
$$

и заметим, что все четыре слагаемых в круглых скобках в рассматриваемом случае неотрицательны. Поэтому

$$
\sum_{k=1}^{n} \sum_{r=n+1}^{2 n} \sum_{l=L-n+1}^{L} \sum_{s=L-n+1}^{L} R\left(h_{\theta}\right)^{-(L-s)} \mathbf{I}\{r+s \leqslant k+l\} \leqslant\left(1-R\left(h_{\theta}\right)^{-1}\right)^{-4},
$$

здесь I $\{\cdot\}$ обозначает индикатор множества в фигурных скобках. Из (15), (16) заключаем, что

$$
\Sigma_{1} \leqslant c_{\theta}^{\prime} \mathbf{P}\left(S_{L} \geqslant \theta L\right)\left(1-R\left(h_{\theta}\right)^{-1}\right)^{-4} \text {. }
$$


Члены суммы $\Sigma_{2}$ оценим с помощью неравенства (3)

$$
\rho(\alpha, \beta, \gamma) \leqslant c p_{\theta}^{L-\beta} \mathbf{P}\left(S_{L} \geqslant \theta L\right), \quad 0<p_{\theta}<1,
$$

где $c$ - постоянная. Выделим в $\Sigma_{2}$ слагаемые, удовлетворяющие условию $\gamma \leqslant \alpha$, и обозначим их сумму через $\Sigma_{2}^{\prime}$; сумму слагаемых с условием $\gamma>\alpha$ обозначим через $\Sigma_{2}^{\prime \prime}$. В случае $\gamma \leqslant \alpha, r+s-k-l \leqslant r-k, s \leqslant l$ воспользуемся представлением

$$
L-\beta=L-(k+l-r)=(L-l)+(r-k)
$$

и заметив, что $\mathbf{I}\{r+s>k+l, s \leqslant l\}=\mathbf{I}\{l-(r-k)<s \leqslant l\}$, получим, что

$$
\sum_{k=1}^{n} \sum_{r=n+1}^{2 n} p_{\theta}^{r-k} \sum_{l=L-n+1}^{L} p_{\theta}^{L-l} \sum_{s=L-n+1}^{L} \mathbf{I}\{r+s>k+l, s \leqslant l\} \leqslant \sum_{i=1}^{\infty} i^{2} p_{\theta}^{i} \sum_{i=0}^{\infty} p_{\theta}^{i}=c_{\theta}^{\prime \prime} .
$$

Таким образом,

$$
\Sigma_{2} \leqslant c c_{\theta}^{\prime \prime} \mathbf{P}\left(S_{L} \geqslant \theta L\right)
$$

Случай $\gamma>\alpha$ почти симметричен случаю $\gamma \leqslant \alpha$, отличие состоит лишь в незначительном изменении области суммирования в соответствующем выражении типа (17). В итоге получим, что

$$
\mathbf{P}\left(A_{11}^{(n)} A_{21}^{(n)}\right) \leqslant \Sigma_{1}+\Sigma_{2}^{\prime}+\Sigma_{2}^{\prime \prime} \leqslant \hat{c}_{\theta} \mathbf{P}\left(S_{L} \geqslant \theta L\right),
$$

где $\hat{c}_{\theta}$ - зависящая от $\theta$ постоянная.

Оценивание вероятностей $\mathbf{P}\left(A_{11}^{(n)} A_{m 1}^{(n)}\right), m>2$, проводится по той же схеме, что и при $m=2$. В итоге получаем, что

$$
\mathbf{P}\left(A_{11}^{(n)} A_{m 1}^{(n)}\right) \leqslant \tilde{c}_{\theta} \mathbf{P}\left(S_{L} \geqslant \theta L\right) \hat{p}_{\theta}^{(m-2) n}, \quad 0<\hat{p}_{\theta}<1, \quad j>2,
$$

где $\tilde{c}_{\theta}$ - зависящая от $\theta$ постоянная. Суммируя оценки (18), (19), получаем, что

$$
\sum_{1 \leqslant i<m \leqslant L / n} \mathbf{P}\left(A_{i 1}^{(n)} A_{m 1}^{(n)}\right) \leqslant b_{\theta} \mathbf{P}\left(S_{L} \geqslant \theta L\right) \sum_{1 \leqslant i<m \leqslant L / n} \hat{p}_{\theta}^{(m-i-1) n} \leqslant b_{\theta}^{\prime} \frac{L}{n} \pi(L ; \theta),
$$

где $b_{\theta}, b_{\theta}^{\prime}-$ зависяшие от $\theta$ постоянные.

Неравенство (6) перепишем в виде

$$
\begin{aligned}
\frac{L}{n} \mathbf{P}\left(A_{11}^{(n)}\right)+\frac{L}{n} \sum_{j=2}^{L / n} \mathbf{P}\left(A_{1 j}^{(n)}\right) & \geqslant \mathbf{P}\left(W_{L, L} \geqslant \theta L\right) \\
& \geqslant \frac{L}{n} \mathbf{P}\left(A_{11}^{(n)}\right)-\sum_{1 \leqslant i<m \leqslant L / n} \mathbf{P}\left(A_{i 1}^{(n)} A_{m 1}^{(n)}\right) .
\end{aligned}
$$

Остается подставить в (21) оценки (9), (12), (20) и провести рассуждения, которые для данной области являются стандартными (см. $[3,5,6])$.

Автор приносит глубокую благодарность В. И. Питербаргу, под руководством которого выполнена эта работа. 


\section{Список литературы}

1. Петров В. В., О вероятностях больших уклонений сумм независимых случайных величин. Теория вероятностей и ее применения (1965) 10, 310-322.

2. Bahadur R. R., Ranga Rao R., On deviations of the sample mean. Ann. Math. Statist. (1960) 31, №4, 1015-1027.

3. Питербарг В. И., О больших скачках случайного блуждания. Теория вероятностей и ее применения (1991) 36, №1, 50-62.

4. Довгалюк В. В., Питербарг В. И., Большие уклонения траекторий пуассоновского процесса. В кн.: Вероятностные прочессы и их приложения. МИЭМ, Москва, 1989, с. 112-117.

5. Питербарг В. И., Козлов А. М., О больших скачках случайного блуждания. Теория вероятностей и ее применения (2002) 47, №4, 803-814.

6. Питербарг В. И., Асимптотические методы в теории гауссовских случайных прочессов и полей. Изд-во МГУ, Москва, 1988.

7. Козлов М. В., О частичных суммах Эрдеша-Реньи: большие уклонения, условное поведение. Теория вероятностей и ее применения (2001) 46, №4, 678-696.

8. Deheuvels P., Devroye L., Limit laws of Erdős-Renyi-Shepp type. Ann. Probab. (1987) 15, №4, 1363-1386.

Статья поступила 20.01.2004. 